\section{"Goodbye Dolly?" The ethics of human cloning}

SIR

John Harris ${ }^{1}$ has written an interesting paper on the ethical debate which has surrounded the cloning of the lamb, Dolly, by adult nuclear transfer from mammary gland cells. In it he rightly attacks the philosophical coherence of the rejection of the principle of cloning for the purposes of human reproduction. Those who do so fall into the trap of providing justifications which were they to be applied universally, would lead - amongst other things - to the abolition of all in vitro fertilisation (IVF) treatment, the condemnation of identical twins to a life of inevitable indignity and the illegality of having children for instrumental purposes for any reasons other than as "ends in the themselves" (which of course means most of them).

In his exposure of the weakness of such arguments Harris appears to endorse research into cloning for reproductive purposes, bidding Dolly a cautious "Hello". While we have no objection to the same conclusion in principle, dramatic moral problems must be faced in squaring such research with an acceptable risk/ benefit ratio for the first resulting cloned human. Although it is impossible properly to calculate the risks to individual participants in any medical research until it is completed, there must be some substantial evidence of an acceptable projection of risks for it to be allowed to start. Thus at some point a decision will have to be made that the risks for the first cloned child will be minimal enough to be at least consistent with the interests of the resulting child. Yet scientifically it is unclear whether we will ever be able to arrive at such a conclusion with enough certainty to allow this first experimental clone to occur.
One mechanism for the production of disease is by somatic mutation of the "healthy" allele at a heterozygous locus. This causes "loss of constitutional heterozygosity" and can predispose individuals in which it occurs to the development of cancers and immunological disease. ${ }^{2}$ Such somatic mutations occur with aging and may in part be due to infidelity of DNA copying during rounds of mitosis. The spontaneous rates of mutation can be very variable depending on the cell type, the locus considered and the previous exposure of the individual to mutational agents (for example, sunlight, ionising radiation, specific chemicals and foodstuffs etc. ${ }^{3}$ ) Mutations can accumulate in nuclei of both somatic and germ-line cells and on the basis of current understanding the accumulation is not negligible and cannot be identified by inspection of a nucleus at any specific time unless the DNA is extracted for analysis of such mutants.

Unlike cloning, one advantage of sexual reproduction, including IVF, is that the zygote now receives a new random assortment of alleles from both parents so that previous somatic mutations from one parent are now paired with alleles of the other. This "dilutes", as it were, the load of somatic mutations from each parent. If unluckily both parents carry recessive disease-related alleles at the same locus then this "dilution" no longer occurs and 1:4 of their offspring are likely to develop the disease. This is more probable if the parents are closely related since they are then more likely to possess similar recessive mutations at identical loci - hence the genetic hazards of consanguineous marriages.

Asexual reproduction bypasses this new assortment of alleles and the acquired somatic mutations of the adult nucleus used for cloning will be transmitted to the next generation where the on-going rate of somatic mutation will produce further loss of heterozygosity. Again this may lead to an increased incidence of cancer, immunological disease and rate of aging. For this reason, without a dramatic breakthrough in genetic analysis, it will remain impossible to calculate the risks of the experimental creation of cloned humans for the individual involved. Yet until this is done, such creation violates all the accepted principles of research ethics, especially since the participating subject is unable in principle to give consent. $^{4}$

Hence the moral paradox of human cloning. The risk is the handicap of a child from birth with a multitude of somatic mutations in the adult nucleus used for transfer. Yet until it is tried, it is unclear how we will ever know the extent of expression of these mutant alleles after introduction into a human gamete. Provided that research on cloning humans remains subject to standard principles of research ethics, it is hard to see how this Catch 22 situation can be overcome. We believe that this argument should figure more prominently in ethico-legal discussions about this fascinating subject, especially since it is the first which must be faced if the creation of the first cloned human is to be allowed at all.

\section{References}

1 Harris J. "Goodbye Dolly?" The ethics of human cloning. Fournal of Medical ethics 1997;23:353-60.

2 Mueller RF, Young ID. Elements of medical genetics [9th ed]. Edinburgh: Churchill Livingstone, 1995: 171-2.

3 Vogel F. Mutation in man. In: Emery AH, Rimoin DL, eds. Principles and practice of medical genetics vol 1, [2nd ed]. Edinburgh: Churchill Livingstone, 1990.

4 Doyal L. Informed consent in medical research. British Medical fournal 1997, 314: 1107-11.

PROFESSOR D J GALTON, DSC, FRCP Department of Human Metabolism and Genetics

PROFESSOR L DOYAL

Department of Human Science and Medical Ethics, St Bartholomew's and the Royal London School of Medicine and Dentistry 